DRAFT VERSION OCTOBER 16, 2018

Preprint typeset using LTEX style emulateapj v. 08/13/06

\title{
ON DIURNAL AND ANNUAL VARIATIONS OF DIRECTIONAL DETECTION RATES OF DARK MATTER
}

\author{
ABHIJIT BANDYOPADHYAY \\ Department of Physics, RKM Vivekananda University, Belur Math, Howrah 711202, India \\ DEBASISH MAJUMDAR \\ Astroparticle Physics and Cosmology Division, Saha Institute of Nuclear Physics, 1/AF Bidhannagar, Kolkata 700064, India \\ Draft version October 16, 2018
}

\begin{abstract}
Direction sensitive direct detection of Weakly Interacting Massive Particles (WIMPs) as dark matter would provide an unambiguous non-gravitational signature of dark matter (DM). The diurnal variation of DM signal due to earth's rotation around its own axis can be a significant signature for galactic WIMPs. Because of particular orientation of earth's axis of rotation with respect to WIMP wind direction, the apparent direction of WIMP wind as observed at a detector can alter widely over a day. In this work we calculate the directional detection rates with their daily and yearly modulations in earth-bound dark matter experiments considering detailed features of the geometry and dynamics of the earth-sun system along with the solar motion in galactic frame. A separate halo model namely the dark disc model other than the usual standard halo model for dark matter halo is also considered and the results for two models are compared. We demonstrate the results for two types of gas detectors namely DRIFT (target material CS2) and NEWAGE (target material CF4) that use Time Projection Chamber techniques for measuring directionality of the recoil nucleus. The WIMP mass and recoil energy dependence of the daily variation of event rates are computed for specific detector and the sensitive ranges of mass and recoil energies for the considered detector are probed.
\end{abstract}

Subject headings: Dark Matter detection, Diurnal and Annual modulations

\section{INTRODUCTION}

Dark matter is a non-luminous, non-baryonic, pervasive fluid extending in and around the bound astronomical systems like galaxy and galaxy clusters. This hypothetical proposition emerged from an attempt to reconcile astronomical observations with Newtonian dynamics. Indirect indications of existence of dark matter is perceived through their putative gravitational interactions in several cosmological observations like rotation curves of spiral galaxies, the gravitational micro-lensing, observations on Virgo and Coma clusters (McLaughlin 1998; Lokas et al. 2003) bullet clusters (Bradac 2009) etc. These observations indicate the existence of roughly spherical halo of dark matter around the visible galaxies. Most of the constituents of dark matter is believed to be non-relativistic (cold dark matter) with Weakly Interacting Massive Particles (WIMPs) as the plausible candidates. The direct detection of the dark matter in terrestrial detectors involves the scattering of the target nuclei in a detector when dark matter interacts with them. The resulting recoil nuclei deposit their energy in the detector to produce dark matter signal in the detector. Dedicated detectors are operational in search of any non-gravitational interaction of dark matter with ordinary matter to provide more insight into the nature of dark matter and its interactions. The dynamics of rotation of the Milky Way galactic disc through the halo of dark matter causes the earth to experience a wind of WIMPs apparently flowing along a direction opposite to that of the motion of the solar system relative to the dark matter halo. There are several ongoing/proposed experimental efforts (Ahmed et al. 2009; Bernabei et al. 2004; Bernabei et al. 2008; Angle et al. 2008; Aprile et al. 2009; Angloher et al. 2008;
Cozzini et al. 2005;

Ninkovic et al. 2005; Burgos et al. 2007; Snowden et al. 2000; Ohnuki et al. 2001; Broniatowski et al. 2009; Sanglard et al. 2005; Tanimori et al. 2004; Rozov et al. 2010 Nishimura et al. 2008; Moulin et al. 2005; $\quad$ Cline et al. 2003; $\quad$ Cline et al. 2001; Bovin et al. 1999; Cebrian et al. 1999; Cebrian et al. 2000; Cebrian et al. 2001; Battat et al. 2009; Sciolla et al. 2008) to directly detect these galactic WIMPs by measuring the energy deposition by the WIMPs through their scattering off the detector nuclei. Unambiguous identification of a WIMP signal is severely challenged by presence of similar background signatures.

An annual modulation is expected (Drukier et al. 1986; Freese et al. 1988) in the detection rates of WIMPs in the direct detection experiments. This seasonal variation is an effect of earth's revolution around the sun in course of which the component of earth's orbital velocity parallel to the WIMP flow direction annually varies causing the earth to encounter different WIMP fluxes at different times of the year. But the relative motion between the sun and the earth is much slower compared to the motion of solar system in galactic halo. Owing to this, the annual modulation is expected to be only a few percent of the WIMP detection rate and it is extremely difficult to extricate it as a positive signature of dark matter from seasonal variation of background rates. On the contrary, observation of directional anisotropy of the WIMP wind on earth is a signature which can hardly be mimicked by any other backgrounds and is potentially more powerful in providing unambiguous signature of galactic WIMPs. There are dedicated experiments with gas detectors with a Time Projection Chamber (TPC) (DRIFT (Burgos et al. 2007; Snowden et al. 2000; Ohnuki et al. 2001), NEWAGE 
(Nishimura et al. 2008; $\quad$ Tanimori et al. 2004), MIMAC (Billard et al. 2009; $\quad$ Richer et al. 2009; Ahlen et al. 2010; $\quad$ Moulin et al. 2005), DMTPC (Battat et al. 2009; Sciolla etal.2008)) or organic crystals (Sekiya et al. 2003) as detectors, aiming to measure the directional (angular) distribution of WIMP induced nuclear recoils. The technique involved in the directional measurements is the 3-D or 2-D reconstruction of nuclear recoil tracks with a good spatial resolution followed by the determination of sense of the recoil direction (discrimination between the head and tail of the recoil track). The directional distribution of the nuclear recoils which carries the imprints of incoming WIMP directions can be exploited to disentangle WIMP signals from backgrounds. ${ }^{1}$. There is a plethora of papers where comprehensive studies of various aspects of directional detection of dark matter has been performed (Green et al. 2008; Green et al. 2007; Copi et al. 2001; Lisanti et al. 2009; Cerdeno et al. 2010; Vergados et al. 2007; Gondolo 2002; Buckland et al. 1994; Lewin et al. 1996; Alenazi et al. 2008; Copi et al. 1999; Copi et al. 2003; Brhlik et al. 1999; Gelmini et al. 2001; Belli et al. 2002; $\quad$ Fornengo et al. 2003; Green 2003; Morgan et al. 2005; Billard et al. 2010; Vergados et al. 2010; Grignon et al. 2010; Creswick et al. 2010). However, because of earth's rotation about its axis which has a particular orientation relative to the direction of incoming WIMPs, the average direction of the WIMP wind with respect to an observer fixed on earth, changes over a sidereal day. Such modulations in the apparent WIMP direction leads to daily fluctuations in the directional (angular) distribution of WIMP signal observed at a static detector on earth. Observation of such diurnal variations in the directional distribution of the WIMP signal would be a robust signature for WIMPs (Spergel 1988; Collar et al. 1992). The directional dependence, however, is sensitive to the velocity distribution of WIMPs in the halo of dark matter. Generally the dark matter halo is considered to be spherical and isothermal (Binney et al. 2008; Lynden-Bell et al. 1967) with the velocity distribution of dark matter WIMPs with respect to the galactic rest frame being Maxwellian which is a steady state solution of the collisionless Boltzmann equation (Chaudhury et al. 2010). Halo model dependence of the directional distribution has been extensively discussed in (Alenazi et al. 2008).

The directional sensitivity is usually realised and presented in terms of galactic coordinates where the description is independent of daily time. In order to comprehend the manifestation of directional sensitivity in the WIMP signal as registered in the earth-bound detectors, it is essential to describe the directional dependence of recoil rates with respect to laboratory (detector) fixed coordinate system. The transformation from galactic frame to laboratory frame naturally invokes temporal variation in the directional description of WIMP rates over a sidereal day. In this work we explicitly calculate the transformation between galactic and laboratory reference frames taking into account details of the earth-sun geometry and dynamics and compute the time dependence of the directional recoil rates in direction sensitive terrestrial dark matter experiments. In order to demonstrate the results of the directional rates calculations in the laboratory frame

1 For a detailed description of directional detection of dark matter see (Sciolla 2009). we assume the standard halo model of dark matter with a Maxwellian velocity distribution for the WIMPs.

In a previous work (Vergados et al. 2009) also the authors have studied the diurnal variation of the direct detection of dark matter in directional experiments. In (Vergados et al. 2009), they express the WIMP velocity in a coordinate system where the polar axis $(Z)$ is in the direction of recoiling nucleus. In the present work, however, the detector fixed coordinate systems are used and the directional detection rates are explicitly computed for two specific detectors. In this case the direction is specified by the direction of recoil nucleus with respect to this detector fixed system. For the study of diurnal variation in (Vergados et al. 2009) the orientation of a detector is specified in the local frame which is given by a specific point in the sky designated by the right ascension $\alpha$ and declination $\delta$. On the contrary, in the present work we explicitly show, for specific detectors, the actual variation of the WIMP detection rates over a day for different recoil directions (of the target nucleus) with respect to the detector fixed frame of reference.

It is also worth investigating the impact if other halo model different from the usual standard halo model is used for these calculations. On the other hand, such investigations may be useful to probe the structure of dark matter halo from the results obtained in actual experiments. In this paper we consider another halo model namely dark disc model (Read et al. 2008) and compare the results with those obtained for standard galactic halo model.

The paper is organised as follows. In Sec. 2 we present the expressions required to calculate the directional recoil rates. Sec. 3 describes the analytic calculations for obtaining the results in laboratory frame of reference. In Sec. 4 we compute the diurnal and annual variation of directional detection rates for two terrestrial dark matter experiments. The response of the detector to the daily variation of event rates for different ranges of WIMP masses and recoil energies is computed in this section. Finally in Sec. 5 we give some concluding remarks.

\section{ANGULAR DEPENDENCE OF THE DIRECT DETECTION RATES}

The differential rate for WIMP induced nuclear recoils per unit detector mass is given by (Alenazi et al. 2008)

$$
\frac{d R}{d E}=\sum_{n} \frac{\rho}{2 \mu_{n}^{2} m} C_{n} \sigma_{n}(E) \mathcal{E}(E) \int_{v>v_{n}} \frac{f(\mathbf{v})}{v} d^{3} v
$$

where, the summation index $n$ represents a particular nuclear species of the target nucleus of the detector (e.g. $n=$ $C, S$ for $C S_{2}$ target in DRIFT detector and $n=C, F$ for $C F_{4}$ target in NEWAGE detector). $C_{n}$ gives the mass fraction of nuclear species $n$ in the target material. In the above $\sigma_{n}(E)=E_{\max }\left(d \sigma_{n} / d E\right),\left(d \sigma_{n} / d E\right)$ being the differential cross section of WIMP-nucleus $(n)$ scattering and $E_{\max }$ is the maximum possible energy transfered between a WIMP and a nucleus of species $n$, which can be expressed in terms of WIMP speed $(v)$, nuclear mass $\left(M_{n}\right)$ and WIMP mass $(m)$ as $E_{\max }=2 \mu_{n}^{2} v^{2} / M_{n}$, with $\mu_{n}=m M_{n} /\left(m+M_{n}\right)$. In this work the local dark matter density $\rho$ is taken to be $0.3 \mathrm{GeV} \mathrm{cm}^{-3}$ and the detection efficiency $(\mathcal{E}(E)$ for recoil energy $E)$ to be $100 \%$. Following (Alenazi et al. 2008) (and references therein), the Radon transformation of the velocity distribution $f(\mathbf{v})$ enables 
one to write the differential rate for WIMP induced nuclear recoils per unit solid angle per unit detector mass as

$$
\frac{d R}{d E d \cos \theta d \phi}=\sum_{n} \frac{\rho}{4 \pi \mu_{n}^{2} m} C_{n} \hat{f}_{n}\left(v_{n}, \mathbf{w}\right) \sigma_{n}(E) \mathcal{E}(E)
$$

The recoil momentum spectrum for nucleus $n$ is denoted by $\hat{f}_{n}\left(v_{n}, \mathbf{w}\right)$ where $\mathbf{w}$ is a unit vector along the direction of nuclear recoil and $v_{n}\left(=c \sqrt{M_{n} E / 2 \mu_{n}^{2}}\right)$ represents the minimum velocity of WIMP required to transfer an amount of energy $E$ to the nucleus $n$ ( $c$ is the speed of light). The function $\hat{f}_{n}\left(v_{n}, \mathbf{w}\right)$ is the 3D Radon transform of the velocity distribution $f(\mathbf{v})$ and is given by $\hat{f}_{n}\left(v_{n}, \mathbf{w}\right)=\int \delta\left(\mathbf{v} \cdot \mathbf{w}-v_{n}\right) f(\mathbf{v}) d^{3} v$ (Alenazi et al. 2008). For a Maxwellian velocity distribution with velocity dispersion $\sigma_{v}$ for the WIMPs, the recoil momentum spectrum in the laboratory rest frame is given by

$$
\hat{f}_{n}\left(v_{n}, \mathbf{w}\right)=\frac{1}{\left(2 \pi \sigma_{v}^{2}\right)^{1 / 2}} \exp \left[-\frac{\left(v_{n}-\mathbf{w} \cdot \mathbf{V}\right)^{2}}{2 \sigma_{v}^{2}}\right]
$$

where, $\mathbf{V}$ is the average velocity of the WIMPs with respect to the detector. The direction of nuclear recoil in three dimension is described in a chosen frame of reference by two angles $\theta$ and $\phi$ as shown in Fig. 1. The recoil energy-integrated di-

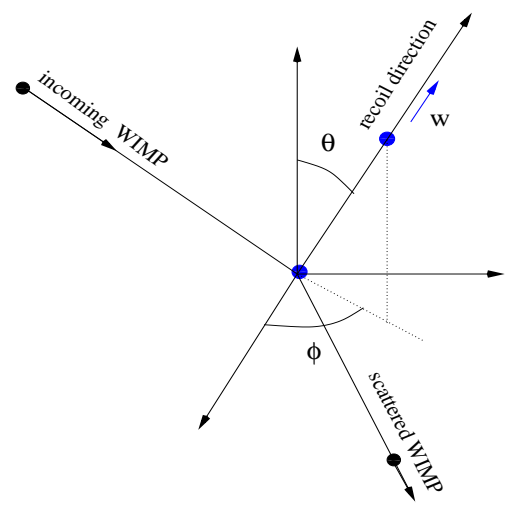

FIG. 1.- Specification of direction of nuclear recoil in WIMP-nucleus scattering.

rectional differential rate is then given by

$$
\frac{d R}{d \cos \theta d \phi}=\int_{E_{\mathrm{th}}} d E\left(\frac{d R}{d E d \cos \theta d \phi}\right)
$$

$E_{\text {th }}$ being the detector threshold energy.

The directional dependence of the observed recoil rates convolves the WIMP velocity distribution in the dark matter halo and average velocity of the WIMPs with respect to the detector. The direction sensitivity of recoil rates are described here, in terms of the two angles $\theta$ and $\phi$, defined in the rest frame of the detector. Hence our computation of expression in Eq. 4 directly gives the angular dependence of rates as actually expected to be observed in a terrestrial detector. In a detector-fixed frame of reference the velocity of the WIMP wind acquires a time-dependent description due to earth's revolution around the sun and earth's rotation about its own axis. As a result the observed angular dependence of the recoil rates, described in the frame of the terrestrial detector, would exhibit both annual and diurnal variations. We compute such annual and diurnal variations of the angular distribution of the event rates in direction sensitive dark matter detectors.

\section{CALCULATION OF $\mathbf{W} \cdot \mathbf{V}$}

In this section we present the analytic calculations of the direction sensitive term $\mathbf{w} \cdot \mathbf{V}$ (Eq. 3 ) in terms of the angles $\theta$ and $\phi$ that specify the recoil direction $\mathbf{w}$ in a terrestrial dark matter detector. We describe the direction sensitivity in a laboratory fixed frame of reference $S_{\mathrm{Lab}}$, denoted in terms of a right-handed system $\left(\mathbf{e}_{\mathbf{L a b} 1}, \mathbf{e}_{\mathbf{L a b 2} 2}, \mathbf{e}_{\mathbf{L a b 3}}\right)$, which are unit vectors pointing respectively towards east, north and vertical direction in the sky at the detector location. As shown in Fig. 2 , a particular direction of nuclear recoil, denoted by the unit vector $\mathbf{w}$, can be expressed in terms of its components in the reference frame $S_{\text {Lab }}$ as

$$
\mathbf{w}=\sin \theta \cos \phi \mathbf{e}_{\mathbf{L a b} 1}+\sin \theta \sin \phi \mathbf{e}_{\mathbf{L a b} 2}+\cos \theta \mathbf{e}_{\mathbf{L a b} 3}
$$

The average velocity $\mathbf{V}$ of WIMP with respect to detector can be written as (Alenazi et al. 2008)

$$
\mathbf{V}=\mathbf{V}_{\mathbf{W G}}-\mathbf{V}_{\mathbf{S G}}-\mathbf{V}_{\mathbf{E S}}-\mathbf{V}_{\mathbf{L E}}
$$

where, $\mathbf{V}_{\mathbf{W G}}$ is the velocity of WIMP with respect to the galactic centre. In standard halo model $\mathbf{V}_{\mathbf{W G}}=0$. $\mathbf{V}_{\mathbf{S G}}$ is the velocity of sun relative to the galactic centre which points towards the Cygnus constellation. $\mathbf{V}_{\mathbf{E S}}$ is the velocity of the centre of mass of the earth relative to the sun and $\mathbf{V}_{\mathbf{L E}}$ is the velocity of the laboratory (detector) relative to the centre of mass of the earth. In the following subsections we present explicit calculations for the quantities $\mathbf{w} \cdot \mathbf{V}_{\mathbf{L E}}, \mathbf{w} \cdot \mathbf{V}_{\mathbf{E S}}$ and $\mathbf{w} \cdot \mathbf{V}_{\mathbf{S G}}$. In obtaining the analytical expressions we have neglected the small eccentricity (0.0167) of the Earth's orbit around the sun.

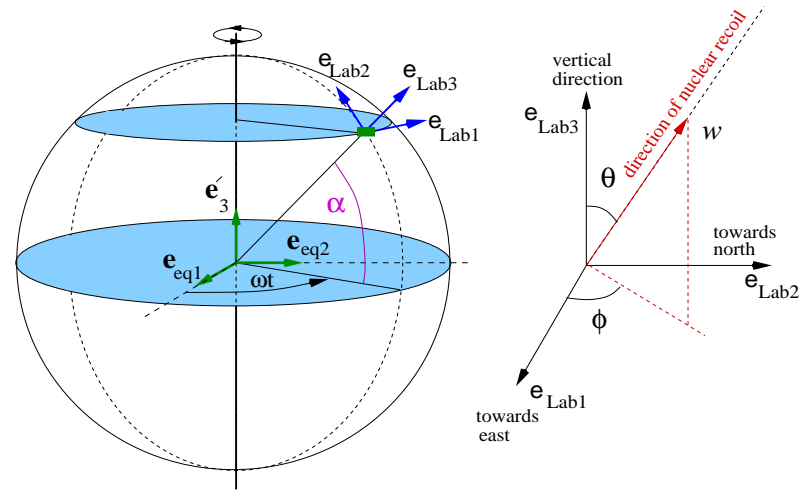

FIG. 2.- Illustration of Laboratory fixed reference frame $S_{\mathrm{Lab}}$ and the direction of nuclear recoil.

\subsection{Expression for $\mathbf{w} \cdot \mathbf{V}_{\mathbf{L E}}$}

The velocity of the detector with respect to the centre of the mass of the earth is along the direction of $\mathbf{e}_{\mathbf{L a b 1}}$ and can be written as

$$
\mathbf{V}_{\mathbf{L E}}=\left|\mathbf{V}_{\mathbf{L E}}\right| \mathbf{e}_{\mathbf{L a b} 1}=\omega R_{\oplus} \cos \alpha \mathbf{e}_{\mathbf{L a b} 1}
$$

$R_{\oplus}$ being the radius of earth and $\omega(=2 \pi / 24 \mathrm{rad} /$ hour $)$ is the angular speed of earth's rotation about its axis and $\alpha$ is the latitude of the detector location. Therefore

$$
\mathbf{w} \cdot \mathbf{V}_{\mathbf{L E}}=\omega R_{\oplus} \cos \alpha \sin \theta \cos \phi
$$

\subsection{Expression for $\mathbf{w} \cdot \mathbf{V}_{\mathbf{E S}}$}

To find $\mathbf{w} \cdot \mathbf{V}_{\mathbf{E S}}$ we bring in three different frames of references: $S_{e q}, S_{1 \odot}$ and $S_{2 \odot}$ which are chosen as follows. The 
frame $S_{e q}$, as shown in Fig. 2, has a origin fixed at the centre of mass of earth and is described by a right-handed system of orthonormal vectors $\left(\mathbf{e}_{\mathbf{e q} 1}, \mathbf{e}_{\mathbf{e q 2}}, \mathbf{e}_{\mathbf{3}}^{\prime}\right)$. The plane spanned by the unit vectors $\mathbf{e}_{\mathbf{e q} 1}$ and $\mathbf{e}_{\mathbf{e q} 2}$ defines the plane of the equator with $\mathbf{e}_{\text {eq1 }}$ pointing vertically upward direction at equator at 12 midnight. $\mathbf{e}_{\mathbf{3}}^{\prime}$ is a unit vector pointing towards direction of angular velocity of earth's rotation and therefore defines the earth's axis of rotation. The transformation between the frames $S_{\mathrm{Lab}}$ and $S_{e q}$ frame is given by

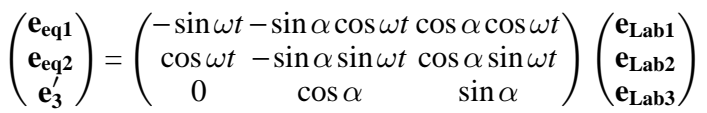

where, $t$ is the time elapsed after 12 midnight. $S_{1 \odot}$ and $S_{2 \odot}$ are two frames of reference with their origins fixed at the sun and are shown in Fig. 3. They are described respectively by the two right-handed systems $\left(\mathbf{e}_{\mathbf{1}}, \mathbf{e}_{2}, \mathbf{e}_{\mathbf{3}}\right)$ and $\left(\mathbf{e}_{\mathbf{1}}^{\prime}, \mathbf{e}_{\mathbf{2}}, \mathbf{e}_{\mathbf{3}}^{\prime}\right)$ where, $\mathbf{e}_{1}, \mathbf{e}_{2}$ are mutually perpendicular unit vectors in the ecliptic plane, with $\mathbf{e}_{\mathbf{1}}$ pointing towards the centre of mass of earth at summer solstice from the sun. $\mathbf{e}_{3}$ is a unit normal to the ecliptic pointing towards the orbital angular velocity of earth. The axis of earth's rotation for all time of the year is parallel to $\mathbf{e}_{1}-\mathbf{e}_{3}$ plane making an angle $\delta=23.5^{\circ}$ with the $\mathbf{e}_{3}$ and at summer solstice its tilt is towards sun. The mutually orthogonal unit vectors $\mathbf{e}_{1}^{\prime}$ and $\mathbf{e}_{2}$ define the plane of the equator. The transformation between frames $S_{1 \odot}$ and $S_{2 \odot}$ is then given by
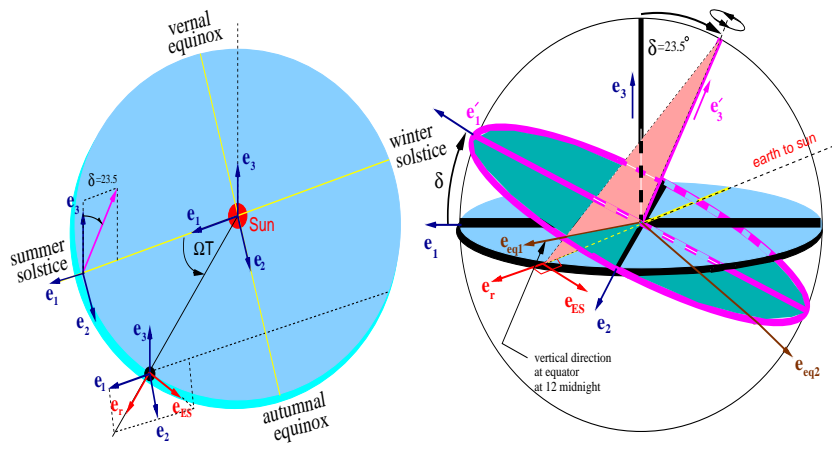

FIG. 3.- Illustration of reference frames $S_{1 \odot}, S_{2} \odot$ and $S_{\text {eq }}$ (described in text)

$$
\left(\begin{array}{l}
\mathbf{e}_{\mathbf{1}} \\
\mathbf{e}_{\mathbf{2}} \\
\mathbf{e}_{\mathbf{3}}
\end{array}\right)=\left(\begin{array}{ccc}
\cos \delta & 0-\sin \delta \\
0 & 1 & 0 \\
\sin \delta & 0 & \cos \delta
\end{array}\right)\left(\begin{array}{l}
\mathbf{e}_{\mathbf{1}}^{\prime} \\
\mathbf{e}_{\mathbf{2}} \\
\mathbf{e}_{\mathbf{3}}^{\prime}
\end{array}\right)
$$

A unit vector along the radius vector of earth with respect to the sun at a time $T$ of the year (counted from the day of summer solstice) is given by

$$
\begin{aligned}
\mathbf{e}_{\mathbf{r}} & =\cos \Omega T \mathbf{e}_{\mathbf{1}}+\sin \Omega T \mathbf{e}_{\mathbf{2}} \\
& =\cos \Omega T \cos \delta \mathbf{e}_{\mathbf{1}}^{\prime}+\sin \Omega T \mathbf{e}_{\mathbf{2}}-\cos \Omega T \sin \delta \mathbf{e}_{\mathbf{3}}^{\prime}
\end{aligned}
$$

where, $\Omega=(2 \pi$ radians/year $)$ is the orbital angular speed of the earth about sun. Since $\mathbf{e}_{\text {eq1 }}$ is along the vertical upward direction at equator at 12 midnight it is parallel to the line of intersection of two planes: the plane containing the axis of the rotation of earth $\left(\mathbf{e}_{\mathbf{3}}^{\prime}\right)$ and the radius vector joining sun to centre of earth and the plane of the equator. The normal to the two planes: the plane containing the vectors $\mathbf{e}_{\mathbf{3}}^{\prime} \& \mathbf{e}_{\mathbf{r}}$ and the plane of the equator respectively are $\mathbf{e}_{\mathbf{3}}^{\prime} \times \mathbf{e}_{\mathbf{r}}$ and $\mathbf{e}_{\mathbf{3}}^{\prime}$. The perpendicular to both of these normals define the line of intersection of the two planes and along which the unit vector is

$$
\begin{aligned}
\mathbf{e}_{\mathbf{e q} \mathbf{1}} & =\frac{\left(\mathbf{e}_{\mathbf{3}}^{\prime} \times \mathbf{e}_{\mathbf{r}}\right) \times \mathbf{e}_{\mathbf{3}}^{\prime}}{\left|\left(\mathbf{e}_{\mathbf{3}}^{\prime} \times \mathbf{e}_{\mathbf{r}}\right) \times \mathbf{e}_{\mathbf{3}}^{\prime}\right|} \\
& =\frac{\cos \Omega T \cos \delta \mathbf{e}_{\mathbf{1}}^{\prime}+\sin \Omega T \mathbf{e}_{\mathbf{2}}}{\sqrt{\cos ^{2} \Omega T \cos ^{2} \delta+\sin ^{2} \Omega T}}
\end{aligned}
$$

The unit vector $\mathbf{e}_{\mathbf{e q} 2}$ is then obtained as

$$
\begin{aligned}
\mathbf{e}_{\mathbf{e q} 2} & =\mathbf{e}_{\mathbf{3}}^{\prime} \times \mathbf{e}_{\mathbf{e q} 1} \\
& =\frac{-\sin \Omega T \mathbf{e}_{1}^{\prime}+\cos \Omega T \cos \delta \mathbf{e}_{\mathbf{2}}}{\sqrt{\cos ^{2} \Omega T \cos ^{2} \delta+\sin ^{2} \Omega T}}
\end{aligned}
$$

Therefore the transformation between reference frames $S_{2 \odot}$ and $S_{e q}$ is given by

$$
\left(\begin{array}{l}
\mathbf{e}_{\mathbf{1}}^{\prime} \\
\mathbf{e}_{\mathbf{2}} \\
\mathbf{e}_{\mathbf{3}}^{\prime}
\end{array}\right)=\left(\begin{array}{ccc}
y \cos \Omega T \cos \delta & -y \sin \Omega T & 0 \\
y \sin \Omega T & y \cos \Omega T \cos \delta & 0 \\
0 & 0 & 1
\end{array}\right)\left(\begin{array}{c}
\mathbf{e}_{\mathbf{e q 1}} \\
\mathbf{e}_{\mathbf{e q 2}} \\
\mathbf{e}_{\mathbf{3}}^{\prime}
\end{array}\right)
$$

where

$$
y=\frac{1}{\sqrt{\cos ^{2} \Omega T \cos ^{2} \delta+\sin ^{2} \Omega T}}
$$

Using Eqs. $(9,10,14)$ we can write the transformation equation between the frames $S_{1 \odot}$ and $S_{\mathrm{Lab}}$ as

$$
\begin{aligned}
& \left(\begin{array}{l}
\mathbf{e}_{1} \\
\mathbf{e}_{2} \\
\mathbf{e}_{3}
\end{array}\right)=\left(\begin{array}{ccc}
\cos \delta & 0-\sin \delta \\
0 & 1 & 0 \\
\sin \delta & 0 & \cos \delta
\end{array}\right)\left(\begin{array}{ccc}
y \cos \Omega T \cos \delta & -y \sin \Omega T & 0 \\
y \sin \Omega T & y \cos \Omega T \cos \delta & 0 \\
0 & 0 & 1
\end{array}\right)
\end{aligned}
$$

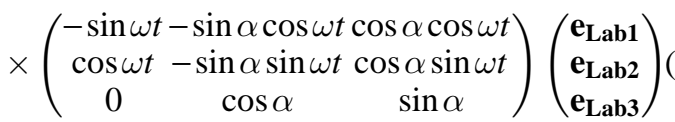

A unit vector along the direction of instantaneous orbital velocity of earth with respect to sun at time $T$ of the year is given by

$$
\mathbf{e}_{\mathrm{ES}}=-\sin \Omega T \mathbf{e}_{\mathbf{1}}+\cos \Omega T \mathbf{e}_{2}
$$

Using the transformation in Eq. 16 we can express it in the laboratory frame of reference as

$$
\begin{aligned}
\mathbf{e}_{\mathbf{E S}}= & y\left(\cos \delta \cos \omega t-\frac{1}{2} \sin 2 \Omega T \sin ^{2} \delta \sin \omega t\right) \mathbf{e}_{\mathbf{L a b} 1} \\
& +(\cos \alpha \sin \delta \sin \Omega T-y \sin \alpha \cos \delta \sin \omega t \\
& \left.-\frac{y}{2} \sin \alpha \sin 2 \Omega T \sin ^{2} \delta \cos \omega t\right) \mathbf{e}_{\mathbf{L a b} 2} \\
& +(\sin \alpha \sin \delta \sin \Omega T+y \cos \alpha \cos \delta \sin \omega t \\
& \left.+\frac{y}{2} \cos \alpha \sin 2 \Omega T \sin ^{2} \delta \cos \omega t\right) \mathbf{e}_{\mathbf{L a b 3}}
\end{aligned}
$$

Finally we get

$$
\begin{aligned}
\mathbf{w} \cdot \mathbf{V}_{\mathbf{E S}}= & \left|\mathbf{V}_{\mathbf{E S}}\right|\left(\mathbf{w} \cdot \mathbf{e}_{\mathbf{E S}}\right) \\
= & \left|\mathbf{V}_{\mathbf{E S}}\right|\left[y\left(\cos \delta \cos \omega t-\frac{1}{2} \sin 2 \Omega T \sin ^{2} \delta \sin \omega t\right) \sin \theta \cos \phi\right. \\
+ & (\cos \alpha \sin \delta \sin \Omega T-y \sin \alpha \cos \delta \sin \omega t \\
& \left.-\frac{y}{2} \sin \alpha \sin 2 \Omega T \sin ^{2} \delta \cos \omega t\right) \sin \theta \sin \phi \\
+ & (\sin \alpha \sin \delta \sin \Omega T+y \cos \alpha \cos \delta \sin \omega t \\
& \left.\left.+\frac{y}{2} \cos \alpha \sin 2 \Omega T \sin ^{2} \delta \cos \omega t\right) \cos \theta\right]
\end{aligned}
$$

where, we have taken the magnitude $\left|\mathbf{V}_{\mathbf{E S}}\right|$ of earth's orbital speed as $30 \mathrm{~km} / \mathrm{s}$ (Plank 2008) 


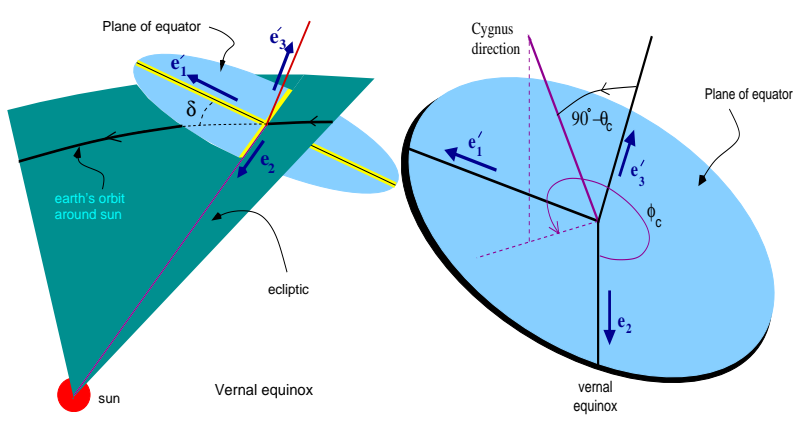

FIG. 4.- Left panel: Relative orientation of ecliptic and plane of equator at the position of vernal (March) equinox. Right panel: Declination and right ascension of Cygnus direction or direction of velocity of sun with respect to galactic centre has been illustrated.

\subsection{Expression for $\mathbf{w} \cdot \mathbf{V}_{\mathbf{S G}}$}

The velocity $\mathbf{V}_{\mathbf{S G}}$ of the sun with respect to the galactic centre happens to point towards the direction of Cygnus constellation. The orientation of Cygnus constellation in celestial coordinate system is given by a declination of $\theta_{c}=42^{\circ}$ and a right ascension of 20.62 hours (Ridpath et al. 2007). This implies that the vector $\mathbf{V}_{\mathbf{S G}}$ makes an angle $90^{\circ}-\theta_{c}$ with the axis of rotation of earth $\left(\mathbf{e}_{\mathbf{3}}^{\prime}\right)$ and its projection on the plane of the equator subtends an angle $\phi_{c}=20.62 \mathrm{~h} \simeq 309^{\circ}$ when measured (anticlockwise) from the direction in sky where the sun crosses the celestial equator at vernal equinox. This direction is defined by $\mathbf{e}_{2}$. Therefore a unit vector along the direction of $\mathbf{V}_{\mathbf{S G}}$ can be written in the frame $S_{2 \odot}:\left(\mathbf{e}_{\mathbf{1}}^{\prime}, \mathbf{e}_{\mathbf{2}}, \mathbf{e}_{\mathbf{3}}^{\prime}\right)$ as

$$
\mathbf{e}_{\mathbf{S G}}=-\cos \theta_{c} \sin \phi_{c} \mathbf{e}_{\mathbf{1}}^{\prime}+\cos \theta_{c} \cos \phi_{c} \mathbf{e}_{\mathbf{2}}+\sin \theta_{c} \mathbf{e}_{\mathbf{3}}^{\prime}
$$

The transformation matrix between the frames of reference $S_{2 \odot}:\left(\mathbf{e}_{1}^{\prime}, \mathbf{e}_{\mathbf{2}}, \mathbf{e}_{\mathbf{3}}^{\prime}\right)$ and $S_{\mathrm{Lab}}:\left(\mathbf{e}_{\mathrm{Lab} 1}, \mathbf{e}_{\mathrm{Lab} 2}, \mathbf{e}_{\mathrm{Lab} 3}\right)$ can be obtained using Eqs. $(9,14)$ as

$$
\begin{aligned}
\left(\begin{array}{l}
\mathbf{e}_{\mathbf{1}}^{\prime} \\
\mathbf{e}_{\mathbf{2}} \\
\mathbf{e}_{\mathbf{3}}^{\prime}
\end{array}\right) & =\left(\begin{array}{ccc}
y \cos \Omega T \cos \delta & -y \sin \Omega T & 0 \\
y \sin \Omega T & y \cos \Omega T \cos \delta & 0 \\
0 & 0 & 1
\end{array}\right) \\
& \times\left(\begin{array}{ccc}
-\sin \omega t-\sin \alpha \cos \omega t \cos \alpha \cos \omega t \\
\cos \omega t & -\sin \alpha \sin \omega t & \cos \alpha \sin \omega t \\
0 & \cos \alpha & \sin \alpha
\end{array}\right)\left(\begin{array}{l}
\mathbf{e}_{\text {Lab1 }} \\
\mathbf{e}_{\mathbf{L a b 2}} \\
\mathbf{e}_{\mathbf{L a b 3}}
\end{array}\right)
\end{aligned}
$$

Exploiting the above equations, $\mathbf{e}_{\mathbf{S G}}$ can be expressed in terms of the laboratory frame of reference as

$$
\begin{aligned}
\mathbf{e}_{\mathbf{S G}}= & (-A \sin \omega t+B \cos \omega t) \mathbf{e}_{\mathbf{L a b 1}} \\
& +\left[-\sin \alpha(A \cos \omega t+B \sin \omega T)+\cos \alpha \sin \theta_{c}\right] \mathbf{e}_{\mathbf{L a b 2}} \\
& +\left[\left(\cos \alpha(A \cos \omega t+B \sin \omega T)+\sin \alpha \sin \theta_{c}\right] \mathbf{e}_{\mathbf{L a b 3}}(22)\right.
\end{aligned}
$$

where,

$$
\begin{aligned}
& A=y \cos \theta_{c}\left(-\sin \phi_{c} \cos \Omega T \cos \delta+\cos \phi_{c} \sin \Omega T\right) \\
& B=y \cos \theta_{c}\left(\cos \phi_{c} \cos \Omega T \cos \delta+\sin \phi_{c} \sin \Omega T\right)
\end{aligned}
$$

Thus we have

$$
\begin{aligned}
\mathbf{w} \cdot \mathbf{V}_{\mathbf{S G}}= & \left|\mathbf{V}_{\mathbf{S G}}\right|\left(\mathbf{w} \cdot \mathbf{e}_{\mathbf{S G}}\right) \\
= & \left|\mathbf{V}_{\mathbf{S G}}\right|\{\sin \theta \cos \phi(-A \sin \omega t+B \cos \omega t) \\
& +\sin \theta \sin \phi\left[-\sin \alpha(A \cos \omega t+B \sin \omega T)+\cos \alpha \sin \theta_{c}\right] \\
& +\cos \theta\left[\left(\cos \alpha(A \cos \omega t+B \sin \omega T)+\sin \alpha \sin \theta_{c}\right]\right\}(.25)
\end{aligned}
$$

Although the physical range of $\left|\mathbf{V}_{\mathbf{S G}}\right|$ is given by $170 \mathrm{~km} / \mathrm{sec}$ $\leq\left|\mathbf{V}_{\text {SG }}\right| \leq 270 \mathrm{~km} / \mathrm{sec}$ (90 \% C.L.) (Leonard et al. 1990; Kochanek 1996; Bovy et al. 2009), in the present work we consider the central value of $\left|\mathbf{V}_{\text {SG }}\right|$ as $220 \mathrm{~km} / \mathrm{s}$.

\section{DIURNAL AND ANNUAL VARIATIONS OF DIRECTIONAL DETECTION RATES}

In this section we calculate the diurnal and annual variations of the direct detection rates with respect to the observed direction of recoil nuclei with the formalism described in the previous sections. In order to estimate such detection rates, the detectors are considered to be direction sensitive, i.e. the directionality of reconstructed tracks of the recoil nuclei are apparent from the observed signals (i.e. the recoil tracks will have no head-tail ambiguity). We also assume the detectors to be $100 \%$ efficient in the present work. From Eq. 2 it is evident that, these rates depend on the target materials and location (latitude) of the detector. For order of magnitude estimations of such rates therefore, the only information of detector properties in this calculation are given in terms of the mass and atomic numbers of the target nuclei and the mass fraction $\left(C_{n}\right)$ of each type of target nucleus (in case of diatomic or multiatomic targets), as required to compute Eq. 2. In this work, we consider two directional detectors namely DRIFT (target material $\mathrm{CS}_{2}$ ) (Burgos et al. 2007; Snowden et al. 2000; Ohnuki et al. 2001) and NEWAGE (target material $\mathrm{CF}_{4}$ ) (Nishimura et al. 2008; Tanimori et al. 2004).

The DRIFT detector at Boulby underground laboratory $\left(54^{\circ} 33^{\prime} \mathrm{N}, 0^{\circ} 49^{\prime} \mathrm{W}\right)$ is designed to detect WIMPs via nuclear recoils within the fiducial volume of the detector. The detector has the ability of not only counting nuclear recoils but also determining the direction of nuclear recoils by reconstructing three dimensional tracks of nuclear recoils. The DRIFT II detector is a Time Projection Chamber (TPC) containing gaseous $\mathrm{CS}_{2}$ at a pressure of 40 torr. It consists of two back to back TPCs on either side of a central photocathode. The ionization electrons resulting from the scattering of WIMPs off the $\mathrm{C}$ or $\mathrm{S}$ atoms of $\mathrm{CS}_{2}$ gas get captured by the electronegative $\mathrm{CS}_{2}$ molecules to form $\mathrm{CS}_{2}^{-}$ions. These ions drift in the electric field towards the anode plate where a multiwire proportional chamber consisting of a two dimensional grid of wires are used as readouts. The $\mathrm{CS}_{2}$ ions experience only thermal diffusion allowing a $3 \mathrm{D}$ reconstruction of the recoil track range and direction. The NEWAGE is also a direction sensitive dark matter search experiment at Kamioka underground laboratory $\left(36^{\circ} 25^{\prime} \mathrm{N}, 137^{\circ} 18^{\prime} \mathrm{E}\right)$. It uses a three dimensional gaseous tracking detector (Micro-TPC) which has Micro Pixel Chamber as its readout. The gaseous microtime projection chamber is filled with $\mathrm{CF}_{4}$ at 152 torr and is read by a two dimensional fine pitch imaging device called $\mu$-PIC consisting of orthogonally formed cathode and anode strips. The details of detector fabrication has been described in (Nishimura et al. 2009). These kind of techniques are used to reconstruct the WIMP induced nuclear tracks in both two and three-dimensions which then enable one to determine the directionality of the recoil track. The recoil energy threshold for the DRIFT experiment can be $\sim$ tens of keV (Plank 2008). The NEWAGE experimental collaboration has mentioned a recoil energy range $\sim 100-400 \mathrm{keV}$ for their WIMP-search analysis. The lower limit of this is set by analysing the background gamma rejection energy (Miuchi et al. 2007). Since the WIMP induced events will be small it is required to have very low threshold for the dark matter detectors albeit 
it increases the unwanted backgroud and unwanted neutron signals. The expected WIMP induced recoil energy range is $\sim 1-50 \mathrm{keV}$. Shielding etc. are required with specific techniques to reduce the unwanted signals/backgrounds. In this work we have presented the results with zero threshold energy for both DRIFT and NEWAGE.

Owing to the apparent directionality of WIMP wind an anisotropy is expected in distribution of nuclear recoils. The directional rates are also expected to exhibit a daily modulation due to rotation of the earth. Such signals of daily modulation can provide an effective background discrimination and thereby producing powerful signature of galactic dark matter WIMPs.

There are two dominant types of WIMP-nucleus elastic scattering - (i) spin-independent interaction, where WIMP interacts with the nuclear mass and (ii) spin-dependent interaction, where the WIMP interaction with the nuclear spin in considered. The WIMP-nucleus scattering cross section can have contributions from either or both of the two interactions. In case of DRIFT detector with target material $\mathrm{CS}_{2}$, only spin-independent interaction contributes to the total scattering cross sections as both $\mathrm{C}$ and $\mathrm{S}$ nuclei have zero spin at the ground state. On the other hand, since F nucleus has a ground state spin $1 / 2$, the NEWAGE detector with target material $\mathrm{CF}_{4}$ is sensitive to both the interactions. But the contribution from the spin-dependent part comes out to be $\sim 2-3$ orders of magnitude higher than that from spin-independent part. Henceforth for NEWAGE we show the event rates with spin-dependent cross sections only.

For a detector with target material $\mathrm{CS}_{2}$, the spinindependent directional recoil rate (as given in Eq. 4) can be written as (Alenazi et al. 2008)

$$
\begin{aligned}
\frac{d R}{d \cos \theta d \phi}= & 1.306 \times 10^{-3} \int d E\left(\frac{\rho}{0.3 \mathrm{GeV}}\right)\left(\frac{\sigma}{10^{-44} \mathrm{~cm}^{2}}\right) \\
& \times \frac{f_{\mathrm{eff}}^{\mathrm{SI}}(E, \mathbf{w})}{4 \pi \mu_{p}^{2} m}\left[\frac{\text { events }}{\mathrm{kg} \cdot \text { day } \cdot \mathrm{sr}}\right] .
\end{aligned}
$$

In the above, $\sigma$ is the WIMP-proton cross section, $\mu_{p}$ is the WIMP-proton reduced mass in $\mathrm{GeV}$ and $f_{\text {eff }}^{\mathrm{SI}}(E, \mathbf{w})$ is given by

$$
f_{\text {eff }}^{\mathrm{SI}}(E, \mathbf{w})=C_{\mathrm{C}} A_{\mathrm{C}}^{2} \hat{f}_{\mathrm{C}}\left(v_{C}, \mathbf{w}\right) \mathcal{F}_{\mathrm{C}}+C_{\mathrm{S}} A_{\mathrm{S}}^{2} \hat{f}_{\mathrm{S}}\left(v_{S}, \mathbf{w}\right) \mathcal{F}_{\mathrm{S}} .
$$

The function $\hat{f}_{\mathrm{C}, \mathrm{S}}\left(v_{C, S}, \mathbf{w}\right)$ can be evaluated using Eq. 3. $\mathcal{F}_{\mathrm{C}}$, $\mathcal{F}_{\mathrm{S}}$ are squares of the nuclear form factors $(F(E))$ given by $F(E)=\exp \left(E / 2 E_{0}\right)$ where $E$ is the energy transferred from WIMP to the target nucleus, $E_{0}=3 /\left(2 m_{N} R_{0}^{2}\right), m_{N}$ being the nuclear mass and $R_{0}$ being the radius of the nucleus (Jungman et al. 1996). The form factor is more accurately given as (Jungman et al. 1996; Engel 1991) $F(E)=$ $\left[\frac{3 j_{1}\left(q R_{1}\right)}{q R_{1}}\right]^{2} \exp \left(-q^{2} s^{2}\right)$, where the momentum transferred $q=$ $\sqrt{2 m_{N} E}, R_{1}=\left(R^{2}-5 s^{2}\right)^{1 / 2}, R \simeq 1.2 A^{1 / 3} \mathrm{fm}$, where $j_{1}$ is a spherical Bessel function, $s=1 \mathrm{fm}$ and $A$ is the mass number of target nucleus. The form factor depends on the distribution of mass and spin within the nucleus and also on the type of the WIMP-nucleus interaction. For very small energy transfer $E$, relevant for our calculation, the form factor $\simeq 1$. $A_{\mathrm{C}}$ and $A_{\mathrm{S}}$ are the mass number of carbon and sulphur nuclei respectively. $C_{\mathrm{S}}$ and $C_{\mathrm{C}}$ are the mass fractions of carbon and sulphur

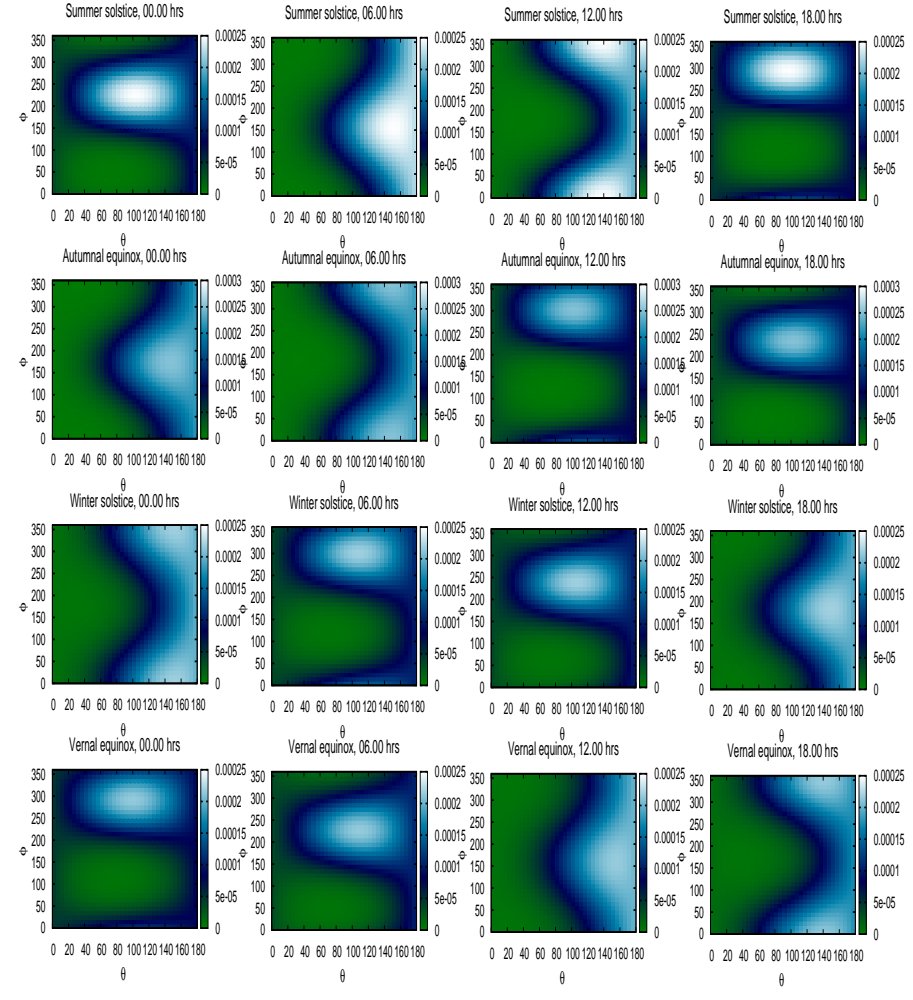

FIG. 5.- These plots shows distribution of energy integrated WIMPnucleon spin-independent event rates in $\mathrm{CS}_{2}$ target (DRIFT) detector over $\theta$ and $\phi$ at different time of the year and at different instants of the day (described in the text). Color contour shows event rates in units of [events/kg/days/sr]. Plots are done with a cross section of $10^{-44} \mathrm{~cm}^{2}$ and a dark matter mass of $60 \mathrm{GeV}$.

respectively and are given by

$$
\begin{aligned}
C_{\mathrm{C}} & =\frac{M_{\mathrm{C}}}{2 M_{\mathrm{S}}+M_{\mathrm{C}}} \\
C_{\mathrm{S}} & =\frac{M_{\mathrm{S}}}{2 M_{\mathrm{S}}+M_{\mathrm{C}}} .
\end{aligned}
$$

In the above $M_{\mathrm{C}}(\approx 11.26 \mathrm{GeV})$ and $M_{\mathrm{S}}(\approx 30.04 \mathrm{GeV})$ are masses of carbon and sulphur nuclei respectively.

The spin-dependent direction recoil rate for a detector with target material $\mathrm{CF}_{4}$ is given by the same expression as Eq. 26 but with $f_{\text {eff }}^{\mathrm{SI}}$ replaced by $f_{\text {eff }}^{\mathrm{SD}}$ which is given by

$$
f_{\text {eff }}^{\mathrm{SD}}=\frac{4}{3} 0.647 C_{F} \hat{f}_{F}\left(v_{F}, \mathbf{w}\right)
$$

where $C_{F}$ is the mass fraction of the fluorine nucleus given by

$$
C_{F}=\frac{4 M_{F}}{4 M_{F}+M_{C}}
$$

$M_{F}$ being the mass $(\approx 17.84 \mathrm{GeV})$ of the fluorine nucleus. The factor (0.647) comes from spin of fluorine nucleus and has been discussed in (Alenazi et al. 2008).

The angular dependence of the spin-independent rates (number of events per day) per $\mathrm{kg}$ of target material $\mathrm{CS}_{2}$ of DRIFT detector are calculated using Eqs. (2-28) . Similar calculations for spin-dependent rates with NEWAGE detector $\left(\mathrm{CF}_{4}\right)$ are performed using Eqs. $(2-26,29-30)$. The results for the spin-independent case (DRIFT) are shown in Fig. 5. The directional rates plotted here are calculated with a benchmark value of WIMP-nucleon cross section of $10^{-44} \mathrm{~cm}^{2}$ and a 


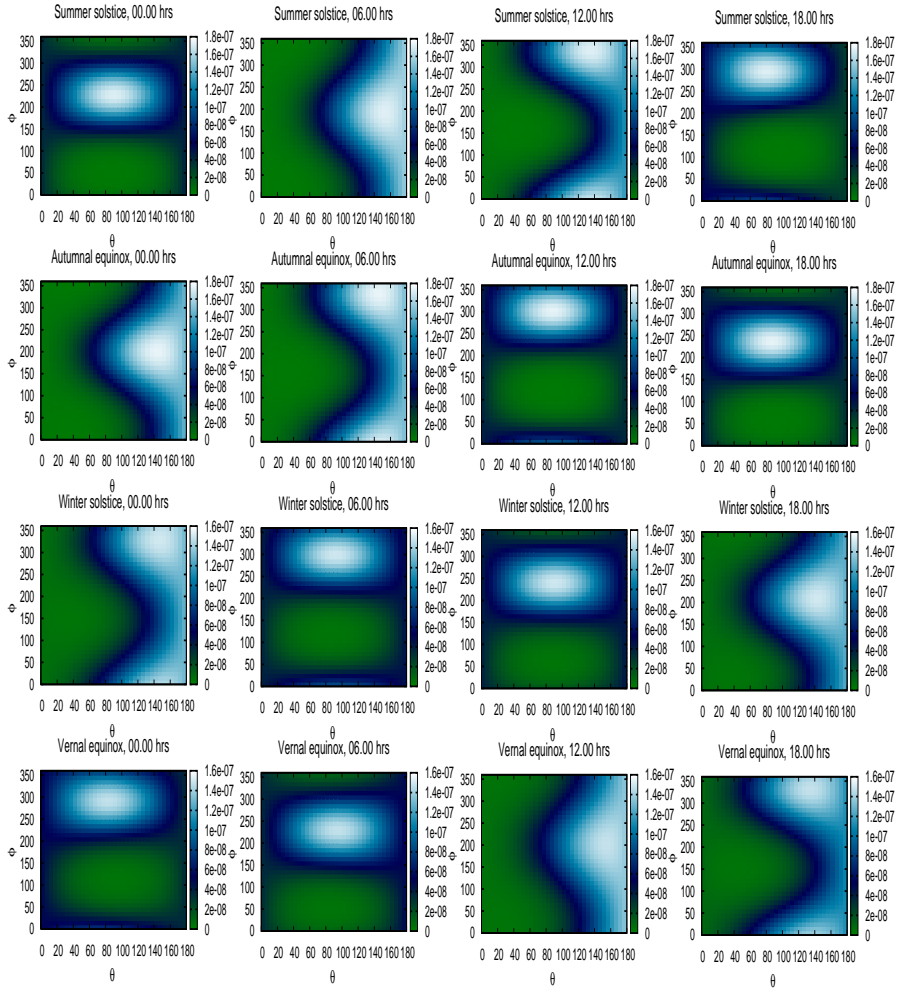

FIG. 6.- Same as Fig. 5 but for spin-dependent events in $\mathrm{CF}_{4}$ target (NEWAGE)

WIMP mass of $60 \mathrm{GeV}$. Each plot shows the variation of event rates with $\theta$ and $\phi$ as defined in Sec. 3. They are shown in Fig. 5 using a colour code symbol. A colour corresponding to a point in the $\theta-\phi$ plane for any single plot in Fig. 5, therefore indicates the detection rate for the directionality given by the $\theta-\phi$ coordinates of that point. The colour codes are chosen such that the increase of detection rates can be represented by the change of colour from green to white in the order green - deep blue - light blue - white. The annual and diurnal variations of the directional dependence of the event rates observed in the detector are illustrated by presenting the plots for different values of the set $(T, t)$, where $T$ and $t$ represent a time of the year and a time in a day respectively. We show the results for 16 representative sets of $(T, t)$ constructed out of four different times of the year namely summer solstice, autumnal equinox, winter solstice and vernal equinox and for four different times of the day $0.00 \mathrm{hrs}$ (midnight), $6.00 \mathrm{hrs}$ (6 A.M.), $12.00 \mathrm{hrs}$ (noon) and $18.00 \mathrm{hrs}$ (6 P.M.). The plots of a given column represent the annual variation of the directional rates observed at a particular time of the day. The plots of a given row, on the other hand, represent the diurnal variations of the directional rates observed at a particular time of the year. For example, from Fig. 5 we see that, for $(\theta, \phi)=\left(100^{\circ}, 230^{\circ}\right)$ the directional rate is $5.5 \times 10^{-6}$ events $/ \mathrm{kg} / \mathrm{days} / \mathrm{sr}$ at 0.00 hours on the day of summer solstice (left corner plot of Fig. 5). As one goes down along the first column of plots (annual variation) in Fig. 5 this rate changes to $4.5 \times 10^{-5}, 1.2 \times 10^{-4}, 2.9 \times 10^{-5}$ in units of events $/ \mathrm{kg} /$ days $/ \mathrm{sr}$ for autumnal equinox, winter solstice and vernal equinox respectively at 0.00 hours. The representative colour for the rates also changes accordingly. This rate also shows the variations in different times of a day for the chosen value of $\theta, \phi$ as one moves row-wise from the left corner plot of Fig. 5. In this particular example the rate varies from $5.5 \times 10^{-6}$ events $/ \mathrm{kg} / \mathrm{days} / \mathrm{sr}$ to $6.5 \times 10^{-5}$,
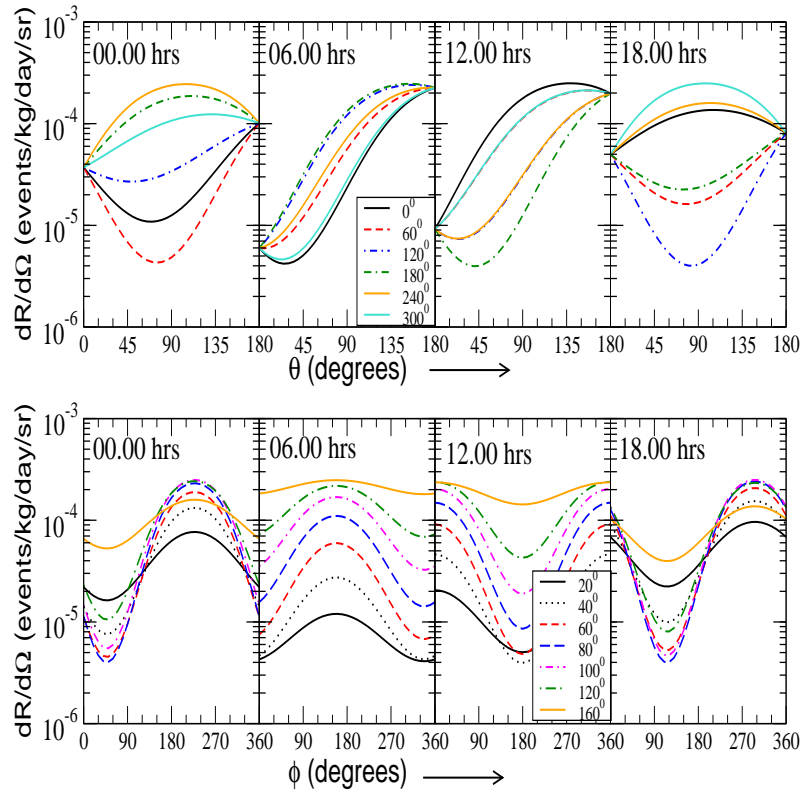

FIG. 7.- upper panel: $\frac{d R}{d \Omega}$ (spin-independent) vs $\phi$ at different values of $\theta$ marked at the inset of $2^{\text {nd }}$ column. Lower panel: $\frac{d R}{d \Omega}$ (spin-independent) vs $\theta$ at different values of $\phi$ marked at the inset of $3^{\text {rd }}$ column. We have chosen a WIMP-nucleon cross section of $10^{-44} \mathrm{~cm}^{2}$. dark matter mass of $60 \mathrm{GeV}$. The target material is $C S_{2}$ (DRIFT detector)
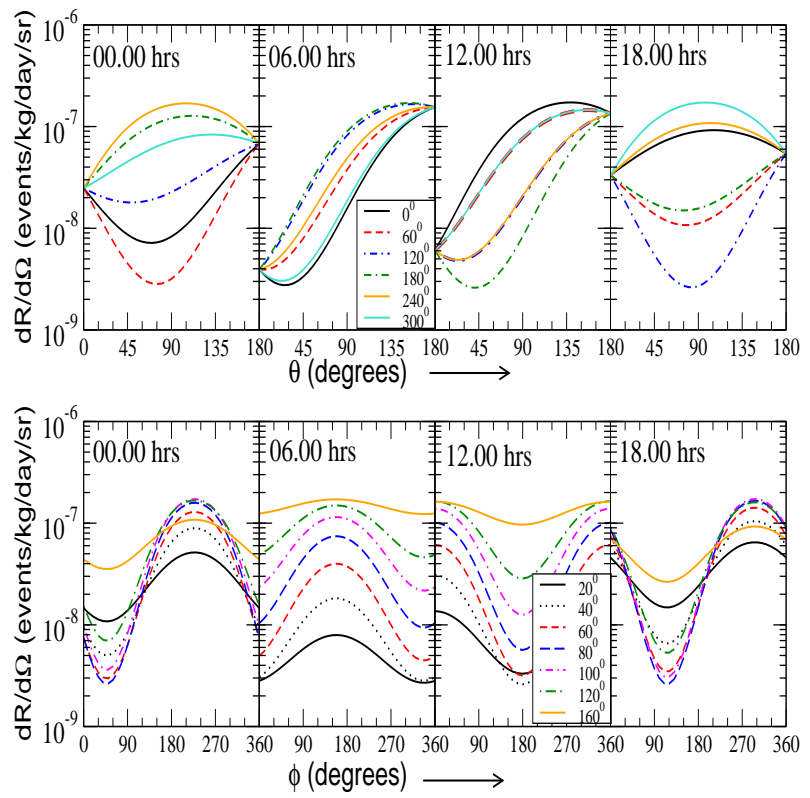

FIG. 8.- Same as Fig. 7 but for spin-dependent interactions at a $\mathrm{CF}_{4}$ in NEWAGE detector.

$1.5 \times 10^{-4}, 2.7 \times 10^{-5}$ (in units of events $/ \mathrm{kg} /$ days $/ \mathrm{sr}$ ) that correspond to three different times of the day namely 6.00 , 12.00 and 18.00 hours respectively on the day of summer solstice. As is evident from Fig. 5, these daily variations of the rates are also indicated by the change of representative colours. Similar observations can be made for plots in any other chosen rows or columns. In Fig. 6 we present the same plots as in Fig. 5 but for spin-dependent events in a $\mathrm{CF}_{4}$ target of NEWAGE detector. The pattern of variation of event rates with $\theta$ and $\phi$ differs in the two figures (Figs. 5 and 
6). From Eqs. (27) and (29) we expect amplitude variation between the spin-dependent and spin-independent case. Again from Eq. (25) along with Eqs. (2) and (3) one sees that the directional rates also depend on the latitude $(\alpha)$ of the detector location ${ }^{2}$. This is reflected in Figs. 5 and 6 . The diurnal variations of angular dependence of the directional rates are also shown for both the cases of spin-independent and spin-dependent WIMP-nucleus interactions in Fig. 7 and Fig. 8 respectively for more clarity. In these two figures we have shown the variations of the directional event rates $d R / d(\cos \theta) d \phi$ with $\theta$ at different values of $\phi$ (upper panel) and with $\phi$ at different values of $\theta$ (lower panel). The plots are shown at four different times of a given day (the summer solstice). From Fig. 7 it is evident that, for $\mathrm{CS}_{2}$ detector, the range of directional variations (variations in $\theta, \phi$ ) of the spin-independent rates lies between $\sim\left(5 \times 10^{-6}-2 \times 10^{-4}\right)$ events per day per unit solid angle per $\mathrm{kg}$ of detector mass. The corresponding range for spin-dependent rates in a $\mathrm{CF}_{4}$ detector is $\left(2 \times 10^{-9}-2 \times 10^{-7}\right)$ events/day/kg/sr ( Fig. 8 ). We also show in Fig. 9 the daily variation of $\theta, \phi$ - integrated rates for DRIFT detector for WIMP mass of $60 \mathrm{GeV}$. The large daily variation is also apparent from this figure.

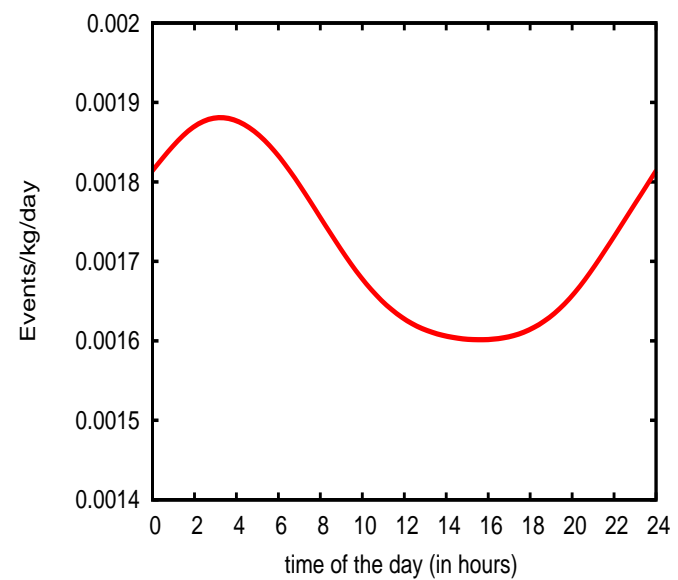

FIG. 9.- Daily variation of $\theta, \phi$ - integrated rates for DRIFT detector for WIMP mass of $60 \mathrm{GeV}$

It is also evident from Figs. 5 - 8 that calculated daily and annual variations of detected number of events within a given solid angle is sensitive to the chosen direction about which the solid angle is considered. For example, event number calculated for DRIFT around the direction specified (in the Laboratory frame) by $\theta=90^{\circ}$ and $\phi=300^{\circ}$ has a larger variation over a day and also over the year. This can be understood from Fig. 5 as the representative colour associated with the point exhibits a span of variations ranging from green to white over different times of a day (or a year). On the contrary, number of events calculated around the direction specified by $\theta=20^{\circ}$ and $\phi=25^{\circ}$ shows mild daily and annual variations. This is realised from Fig. 5 since over different times of a day (or a year) the point lies mostly in the green region of the $\theta-\phi$ plane. The daily variations of direct detection rates for three chosen directions of recoil tracks are shown in Fig. 10 for illustrative purposes. In Fig. 10 we plot the calculated number of events within a

${ }^{2}$ DRIFT is at a latitude $\alpha=54^{\circ} 33^{\prime} \mathrm{N}$, for NEWAGE $\alpha=36^{\circ} 25^{\prime} \mathrm{N}$

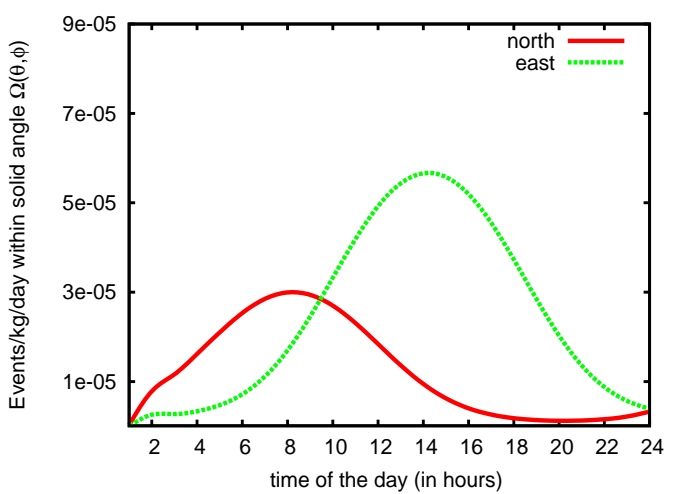

FIG. 10.- The number of nuclear recoils in two solid angles with differently chosen directions namely, north and east in the laboratory frame of reference as a function of time of a day. The plots are shown at the day of summer solstice.

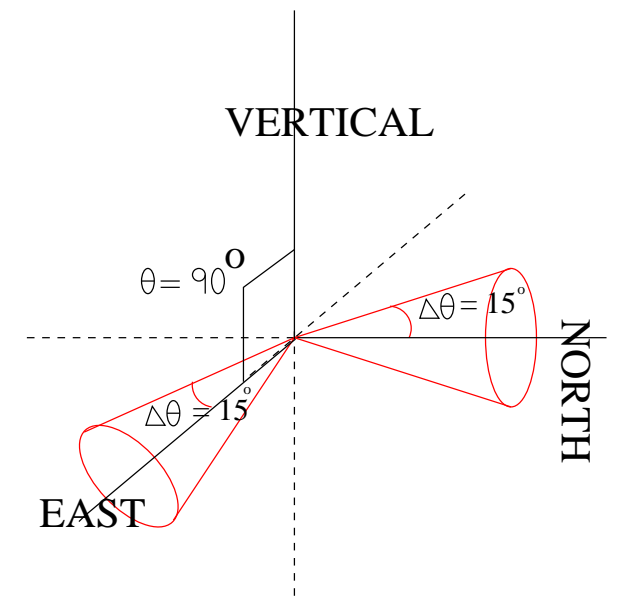

FIG. 11.- Schematic diagram to show the choice of solid angles as described in the text.

solid angle considered about two different chosen directions namely north and east as a function of different times of a sidereal day. In this case we have chosen this day to be the day of summer solstice. The solid angles are so chosen that the semi-vertical angle of the cone around the chosen direction is $15^{\circ}$. It reveals larger daily variations along east direction in comparison to the northern direction for this case. Experiments dedicated to directional dark matter detection may therefore look for some preferential directions as obtained from such theoretical studies for the daily variations calculated in the Laboratory frame of reference, along which the substantial daily variation is expected.

The diurnal variations in the directional distribution of WIMP signals can be better illustrated by studying the daily fluctuations of a dimensionless quantity defined as the ratio of directional recoil rate in a direction $\mathbf{w}_{1}\left(\theta_{1}, \phi_{1}\right)$ to that in a direction $\mathbf{w}_{2}\left(\theta_{2}, \phi_{2}\right)$. In a practical scenario this can be estimated by considering ratio of number of events for two solid angles $\Omega_{1}$ and $\Omega_{2}$ around the two specified directions ( $\mathbf{w}_{1}$ and 
$\mathbf{w}_{2}$ ) which can be defined as

$$
\begin{aligned}
r\left[\mathbf{w}_{1} ; \mathbf{w}_{2}\right] & =\frac{\int_{\Omega_{1}} \frac{d R}{d \Omega} d \Omega}{\int_{\Omega_{2}} \frac{d R}{d \Omega} d \Omega} \\
& =\frac{\int_{\phi_{1}-\Delta \phi}^{\phi_{1}+\Delta \phi} \int_{\theta_{1}-\Delta \theta}^{\theta_{1}+\Delta \theta} d \phi d \theta \sin \theta \frac{d R}{d \Omega}(\theta, \phi)}{\int_{\phi_{2}-\Delta \phi}^{\phi_{2}+\Delta \phi} \int_{\theta_{2}-\Delta \theta}^{\theta_{2}+\Delta \theta} d \phi d \theta \sin \theta \frac{d R}{d \Omega}(\theta, \phi)}
\end{aligned}
$$

Such variations, estimated for the DRIFT detector, are shown in Fig. 12 where we have presented the daily variation of the quantity $r\left[\mathbf{w}_{1}, \mathbf{w}_{2}\right]$ on the day of summer solstice for a representative set: $\left(\mathbf{w}_{1}, \mathbf{w}_{2}\right)$ chosen as $\left\{\right.$ east $\left(\theta=90^{\circ}, \phi=0^{\circ}\right)$, $\left.\operatorname{north}\left(\theta=90^{\circ}, \phi=90^{\circ}\right)\right\}$. with the semi vertical angle of the cones (that define the solid angles considered here) taken to be $15^{\circ}$. The choice of the solid angles are illustrated in Fig. 11 where two choices of solid angles are shown - Our calculations show that the ratio of detected events corresponding to the eastward recoil direction to that corresponding to the recoils along the north varies in the range $\sim 0.001-21$ over a sidereal day on the day of summer solstice.

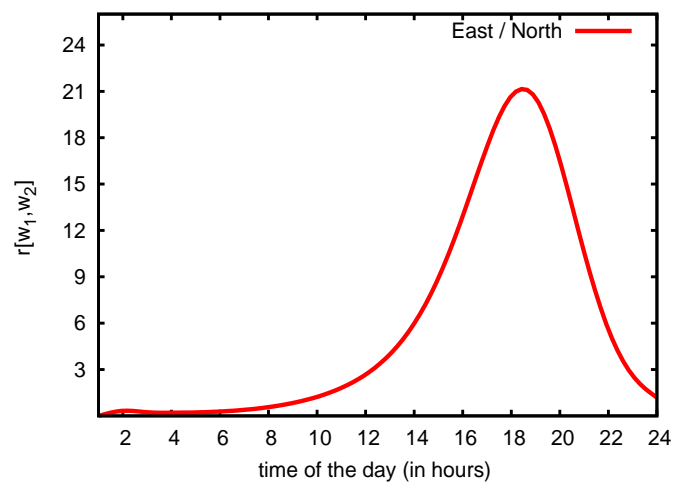

FIG. 12.- The ratio of number of nuclear recoils in two solid angles with differently chosen directions in the laboratory frame of reference as a function of time of a day. The plots are shown on the day of summer solstice. We have presented the ratio for a representative set :( $\left.w_{1}, w_{2}\right)$ : (east, north)

It may be of interest to investigate the daily variation of detection rates as functions of WIMP masses and recoil energies. In Fig. 13 we show the energy integrated rates for different WIMP masses at different time of the day. These rates are plotted for two detectors - DRIFT and NEWAGE considered here. The overall yield for each of the detectors is maximum at WIMP masses around $20 \mathrm{GeV}$. The daily variation also dominates for WIMP masses at around $20 \mathrm{GeV}$ for both the detectors considered here. We also calculate the daily variation of rates with recoil energy for a fixed WIMP mass of $60 \mathrm{GeV}$ and the results are shown in Fig. 14. From Fig. 14 one sees that the overall yield decreases with the increase in recoil energy although the daily variation is more prominent for higher recoil energies. We also investigate the sensitivity of mass ranges to the daily variations for different ranges of recoil energies. For demonstrative purposes we show the results for DRIFT detector only. In Fig. 15 the results are plotted for five fixed values of WIMP masses ranging from $10 \mathrm{GeV}-200 \mathrm{GeV}$. All the rates shown in this figure are integrated rates over $\theta$ and $\phi$ and over the considered energy ranges. The daily variations are investigated for five recoil energy ranges: $0-10 \mathrm{keV}, 5-15 \mathrm{keV}, 10-20$ $\mathrm{keV}, 15-25 \mathrm{keV}, 20-30 \mathrm{keV}$. Beyond $30 \mathrm{keV}$ the rate falls down to very low value. It is evident from Fig. 15 that the

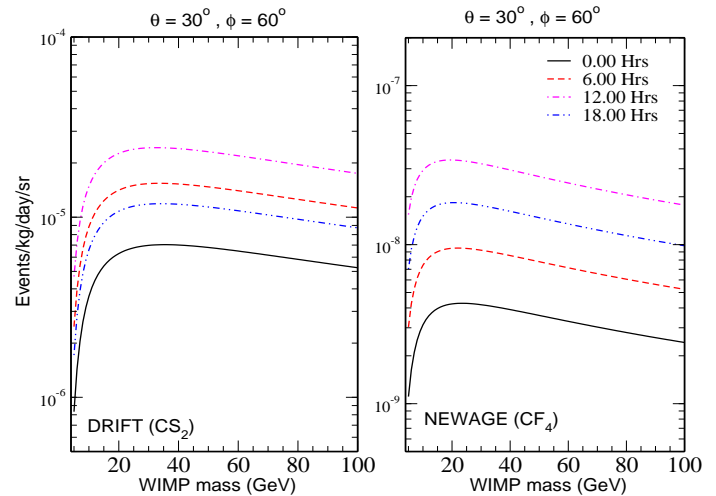

FIG. 13.- Daily variation of detection rates as a function of WIMP mass for DRIFT and NEWAGE detectors.

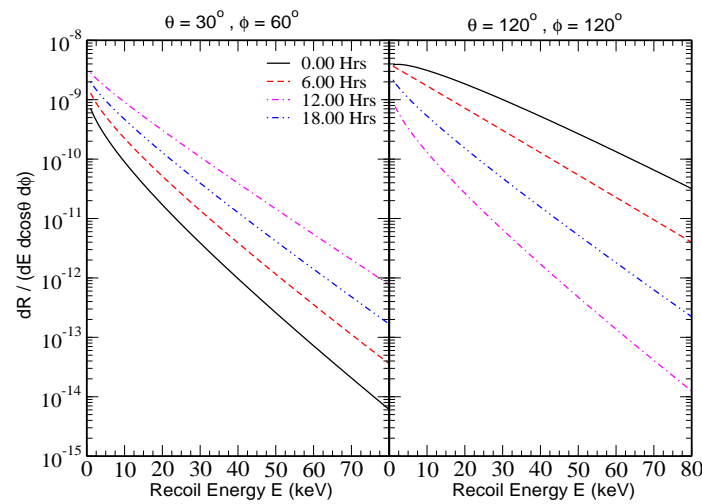

FIG. 14.- Daily variation of detection rates as a function of recoil energy for DRIFT and NEWAGE detectors for a given WIMP mass of $60 \mathrm{GeV}$.

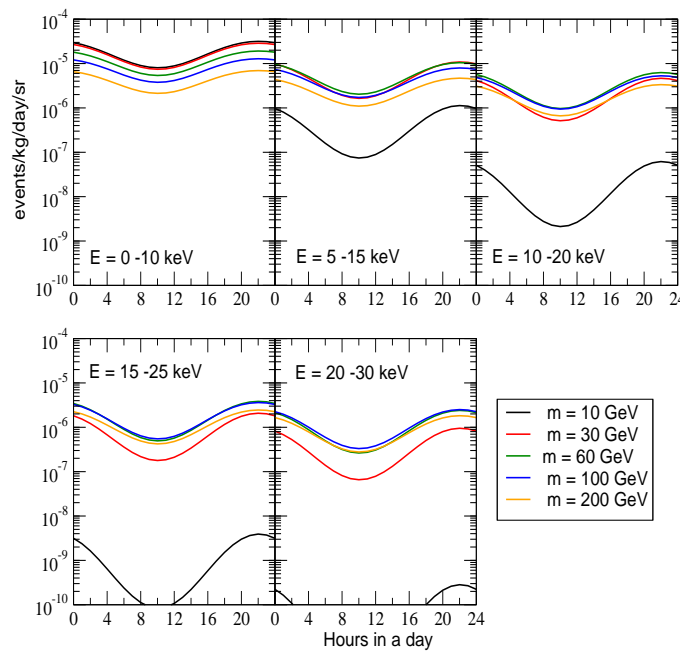

FIG. 15.- Daily variation of detection rates as a function of recoil energy and WIMP mass for DRIFT detector.

overall rate dominates for lower recoil energy range (0-10 $\mathrm{keV}$ ) all along the WIMP mass range considered. The nature of daily variations of the detection rates are also similar for the whole mass range in the recoil energy band of $0-10$ $\mathrm{keV}$. It is also observed from Fig. 15 that for higher recoil energy ranges, the yield for lower WIMP masses gradually decreases but the magnitude of daily variation increases. It 
10

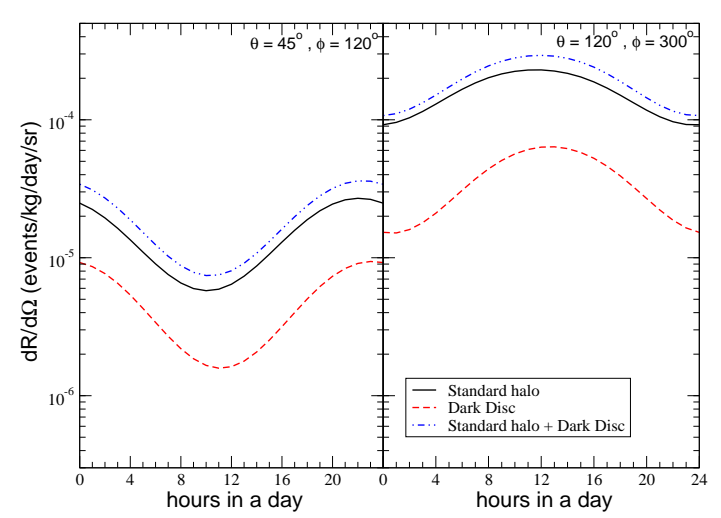

FIG. 16.- Comparison of diurnal variation of directional detection rates for dark disc model and standard halo model for dark matter for two sets of $(\theta, \phi)$ on the day of summer solstice.

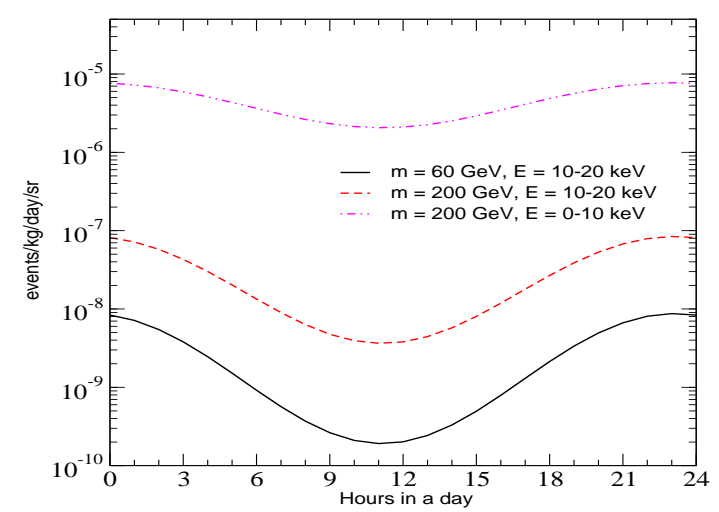

FIG. 17.- Diurnal variation of directional detection rates for dark disc model with different dark matter mass and recoil energy range. The plots are shown for a given $\theta\left(45^{\circ}\right), \phi\left(120^{\circ}\right)$ and on the day of summer solstice

appears from the present calculations that for the particular detector considered, lower recoil energy range is more suited for investigating dark matter if the WIMP masses are having a wide range of values (from very low to very high). But if WIMP masses are limited to only lower values then the daily variation will be more prominent for higher recoil energy ranges.

Diurnal variations of detection rates in alternative galactic halo models: We also calculate the diurnal variations of the directional detection rates considering a different galactic halo model. Such consideration enables us to compare the results obtained from the standard halo model with that from this new model. The actual experimental results when compared with such calculations may throw light on the nature of dark matter halo. To this end, we consider a model proposed by (Read et al. 2008) which is a combination of the usual standard halo model and a proposed dark disc that corotates with the galactic disc. In the dark disc (DD) model it is assumed that the DD kinematics matches with that of Milky Way's thick disc (Bruch et al. 2008). This in turn, gives a rotation lag represented by $\mathbf{V}_{\text {lag }}$ with respect to $\mathbf{V}_{\mathbf{S G}}$ described earlier in Eq. 6 along with a different velocity dispersion. A Maxwellian velocity distribution for dark matter is also considered for dark disc model (Bruch et al. 2008) with $\mathbf{V}_{\mathbf{S G}}$ replaced by $\mathbf{V}_{\text {lag }}$ and in this calculation a dispersion of $50 \mathrm{~km} / \mathrm{s}$ is adopted following (Bruch et al. 2008). In Fig. 16 we show the daily variation of rate on a particular day (summer solstice) for dark disc model and compared them with that of the standard halo model (SHM) for two sets of values of $\theta, \phi$. The rates from the combined model of DD and SHM are also displayed in Fig. 16. From Fig. 16 one sees that the yield in case of standard halo model is more than that for dark disc for the chosen value of dark matter mass. The difference in the yield for the two cases varies when observed in different $\theta, \phi$ directions. The magnitude of daily variations also appears to differ marginally for the two models. However we have checked that this difference between the two models varies for different recoil energies and dark matter mass. In Fig. 17 the diurnal variation for two chosen dark matter masses are shown for different recoil energy ranges considering only the dark disc model. One of the chosen dark matter mass is in the high mass range $(200 \mathrm{GeV})$ and the other one is chosen at 60 $\mathrm{GeV}$ as before. From Fig. 17 one sees that if only dark disc model is considered, the yield is much higher for the higher dark matter mass and low recoil energy range implying that the manifestation of the dark disc effect is more prominant for low recoil energy and large WIMP mass.

\section{CONCLUSION}

Directional detection of dark matter in direct detection experiments not only provides unambiguous signature of galactic WIMPs but also serves as a potentially powerful probe to the structure and dynamics of dark matter halo. The directional detection techniques involve reconstruction and head-tail discrimination of tracks of nuclear recoil, induced by the scattering of WIMPs off the detector nuclei. Directional description of the WIMP induced nuclear recoil rates in terms of angular coordinates defined and realised in the laboratory frame of a detector exhibits temporal variation over a sidereal day due to diurnal motion of the earth. It would also have an annual modulation owing to yearly variations of the component of earth's orbital velocity along incoming WIMP direction. Analytical details of the annual and diurnal variations are determined by the dynamics and geometry of the sun-earth system and the location (latitude) of the detector on the earth. Proper transformation of coordinate systems are also needed to obtain the results in terms of laboratory frame of reference. This is very crucial for any detector based prediction of such directional modulations of dark matter signals. The directional rates of WIMP signal events in directional detectors sensitive to spin independent WIMP-nucleus interactions $\left(\mathrm{CS}_{2}\right.$ target $)$ are estimated for all possible recoil directions and they are found to lie within the range $\sim 10^{-6}-10^{-4}$ per day per steradian per $\mathrm{kg}$ of target mass of the detector. A WIMP mass of $60 \mathrm{GeV}$ and WIMPnucleon cross section of $10^{-44} \mathrm{~cm}^{2}$ have been assumed for all the calculations. Similar estimations for directional detectors with dominant sensitivity to spin-dependent WIMP-nucleus interactions gives the corresponding range in same units as $\sim 10^{-9}-10^{-7}$. The diurnal variations in the laboratory frame description of directional anisotropy of the WIMP induced nuclear recoils amount to a wide daily fluctuations (ranging from 0.3 to 20) of the ratio of WIMP rates corresponding to two chosen orthogonal directions of nuclear recoils (east and north).

Energy integrated rates for different WIMP masses ranging from $0-100 \mathrm{GeV}$ show that the daily variation is most prominent for WIMP masses around $20 \mathrm{GeV}$. The study (with WIMP mass of $60 \mathrm{GeV}$ ) reveals that although the range of daily modulation increases with the increase in recoil energies 
but the drop in overall yield for higher recoil energies makes it difficult for a detector to register this modulation. The effect of varied recoil energy ranges on the integrated (over $\theta, \phi$ and over the considered energy range) yield and their daily modulations at DRIFT detector for a wide range of WIMP masses $(10-200 \mathrm{GeV})$ are also investigated. The results show similar daily modulations for the mass range considered for lower recoil energy range $(0-10 \mathrm{GeV})$. The overall yield is also higher in this recoil energy range. Higher recoil energy ranges, although results in the increase in magnitude of modulation for lower WIMP masses but the overall yield drops down. Hence, if the detector is efficient and precise enough to detect the yield corresponding to higher recoil energy ranges then for lower values of WIMP masses this detector will observe considerable daily modulation of the signal (as shown in Fig. 15).

The results of the analysis are found to differ mostly in terms of the total yield when another model for dark matter halo namely the dark disc (where one considers a corotating disc of dark matter with the galactic disc) model or DD model is considered. However, calculations using a combined model of DD and the usual SHM shows marginal increase in overall yield and also a variation of nature of daily modulations for certain recoil directions.

Acknowledgements: D.M. thanks J D Vergados for some discussions.

\section{REFERENCES}

Ahlen, S. et al, 2010, Int. J. Mod. Phys. A 25, 1

Ahmed, Z. et al [CDMS Collaboration], 2009, Phys. Rev. Lett. 103, 141808

Alenazi, M. S. et al., 2008, Phys. Rev. D 77, 043532

Angloher, G. et al, 2008, arXiv:0809.1829 [astro-ph]

Angloher, G. et al, 2005, Astropart. Phys. 23, 325

Angle, J et al [Xenon Collaboration], 2008, Phys. Rev. Lett. 100, 021303

Aprile, E. et al, 2009, Rev. Mod. Phys.

Battat, B. R. et al., 2009, AIP Conf. Proc. 1182, 276

Belli, P. et al., 2002, Phys. Rev. D 66, 043503

Beltran, B. [for the PICASSO collaboration], 2008, J. Phys.:Conference series 136042080

Bernabei, R. et al. [DAMA collaboration], 2004, Eur. Phys. J. C 56

Bernabei, R. et al. [DAMA collaboration], 2008, Nucl. Instrum. Meth. A 592 , 297

Billard, J. et al, 2009, arXiv:0911.4086 [astro-ph.CO]

Billard, J. et al., 2010, arXiv:1009.5568 [astro-ph.IM]

Binney, J. et al., 2008, Galactic Dynamics, 2nd Edition (Princeton University Press, Princeton, 2008)

Bovin, C. et al., 1999, Nucl. Phys. B (Proc. Suppl.) 7090

Bovy, J. et al., 2009, Astrophys. J. 704, 1704

Bradac, M., 2009, Nucl. Phys. Proc. Suppl. 194, 17

Brhlik, M. et al., 1999, Phys. Lett. B 464, 303

Broniatowski, A. et al, 2009, Phys. Lett. B 681, 305

T. Bruch et al. 2008 PoS IDM2008, 049

Buckland, K. N. et al., 1994, Phys. Rev. Lett. 73, 1067

Burgos, S. et al., 2007, Astropart. Phys. 28, 409

Cebriãąn, S. et al., 1999, Astroparticle Physics 10361

Cebriãąn, S. et al., 2000, Nucl. Instr. Meth A 444315

CebriÃąn, S. et al., 2001, Astroparticle Physics 1579

Cerdeno, D. G. et al., 2010, arXiv:1002.1912 [astro-ph.CO]

Chaudhury, S. et al., 2010, JCAP 1009, 020

Cline, D. B. et al, 2003, in Proceedings of 4th International Workshop on the Identification of Dark Matter held at St. William's College, York Minster, York, England, September 2 - September 6, 2002, edited by N. J. C. Spooner (World Scientific 2003).

Cline, D. B. et al, 2001, in Proceedings of third international conference on dark matter and astro- and particle physics held at Heidelberg, Germany, July 10 - 16, 2000, edited by H. V. Kapdor-Kleingrothaus (Springer-Verlag Berlin Heidelberg 2001).

Collar J. I. et al, 1992, Nucl. Phys. Proc. Suppl. 28A, 297

Copi, C. J. et al., 1999, Phys. Lett. B 461, 43

Copi, C. J., 2001, Phys. Rev. D 63, 043507

Copi, C. J. et al., 2003, Phys. Rev. D 67, 103507

Cozzini, C. et al, 2005, Phys. Rev. C 70, 064606

Creswick, R. J. et al., 2010, arXiv:1007.0214 [astro-ph.IM]

Drukier, A.K. et al., 1986 Phys. Rev. D 33, 3495
Read, J. I. et al., 2008, [arXiv:0803.2714 [astro-ph]]

Engel, J., 1991, Phys. Lett. B 264114

Fornengo, N. et al., 2003, Phys. Lett. B 576, 189

Freese, K. et al., 1988, Phys. Rev. D 37, 3388

Gelmini, G. et al., 2001, Phys. Rev. D 64, 023504

Gondolo, P., 2002, Phys. Rev. D 66, 103513

Green, A. M., 2003, Phys. Rev. D 63, 043005

Green, A. M. et al., 2007, Astropart. Phys. 27, 142

Green, A. M. et al., 2008, Phys. Rev. D 77, 027303

Grignon, C. et al., 2010, arXiv:1008.4712 [astro-ph.IM]

Jungman, G. et al., 1996, Physics Reports 267, 195

Kochanek, C. S., 1996, Astrophys. J. 457, 228

Leonard, P. J. T. et al., 1990, Astrophys. J. 353, 486

Lewin, J. D. et al., 1996, Astropart. Phys. 6, 87

Lisanti, M. et al., 2009, arXiv:0911.1997 [hep-ph]

Lokas, E. L. et al., 2003, Mon. Not. Roy. Astron. Soc. 343401

Lynden-Bell, D. et al., 1967, Mon. Not. Roy. Astron. Soc. 136, 101

McLaughlin, D. E, 1998, arXiv:astro-ph/9812242

Miuchi, K. et al., 2007, Phys. Lett. B 654, 58

Morgan, B. et al., 2005, Phys. Rev. D 71, 103507

Moulin, E. et al, 2005, Phys. Lett. B 614, 143

Ninkovic, J. et al, 2006, Nucl. Instrum. Meth. A 564, 567

Nishimura, H. et al, 2008, J. Phys. Conf. Ser. 120, 042025

Nishimura, H. et al., 2009, Astropart. Phys. 31, 185

Ohnuki, T. et al, 2001, Nucl. Instrum. Meth. A 463, 142

Plank, S. J. S., 2008, Ph.D. thesis, The University of Edinburgh, (unpublished)

Richer, J. P. et al, 2009, arXiv:0912.0186 [astro-ph.IM]

Ridpath, I. et al., 2007, Stars and Planets Guide, Princeton University Press, Princeton

Rozov, S. et al, 2010, arXiv:1001.4383 [astro-ph.IM]

Rubbia, A., 2006, J. Phys.:Conference series 39129

Sanglard, V. et al [The EDELWEISS Collaboration], 2005, Phys. Rev. D 71, 122002

Sciolla, G et al., 2008, PoS IDM2008, 002

Sciolla, G., 2009, Mod. Phys. Lett. A 24

Sekiya, H. et al.,2003, Phys. Lett. B 571, 132

Snowden-Ifft, D. P., et al, 2000, Phys. Rev. D 61, 101301

Spergel, D. N., 1988, Phys. Rev. D 37, 1353

Tanimori, T. et al, 2004, Phys. Lett. B 578, 241

Vergados, J. D. et al., 2007, Phys. Rev. D 75, 055007

Vergados, J. D. et al., 2010, arXiv:1009.0631 [astro-ph.CO]

Vergados, J. D. et al., 2009, arXiv:0912.3121 [astro-ph.CO] 